\title{
High environmental compatibility photopolymers compared to PVA/AA based materials at zero spatial frequency limit
}

\author{
S. Gallego ${ }^{1,2}$, A. Márquez ${ }^{1,2}$, M. Ortuño ${ }^{1,2}$, S. Marini ${ }^{1,2}$, J. Francés $^{1}$ \\ ${ }^{1}$ Dept. Física Ingeniería de Sistemas y Teoría de la Señal, Universitat d’Alacant (Spain) \\ Apartado 99 E-03080 Alacant \\ 2 I.U. Física Aplicada a las Ciencias y las Tecnologías Apartado 99 E-03080 Alacant \\ (Spain) \\ Tel.: +34-96-5903400 (ext. 3327); Fax: +34-96-5909750; \\ *Corresponding author: sergi.gallego@ua.es
}

\begin{abstract}
In holographic applications the direct parameters determination of photopolymers as optical recording media is a very difficult task due to the presence of two different phenomena: polymer formation and monomer diffusion. We propose a direct method based on zero spatial frequency recording, to eliminate the diffusion influence, and on interferometric techniques, both in transmission and in reflection, to obtain quantitative values of: shrinkage, polymerization rate, polymer refractive index and relation between polymerization and recording intensity. Recent investigations confirm the toxic potential of acrylamide. Starting from polyvinylalcohol/acrylamide photopolymer we have proposed different compositions of new competitive photopolymers with high environmental compatibility. We have studied the ways to optimize the optical behavior
\end{abstract}


and the environmental compatibility. Parameters comparison with the polyvinylalcohol/acrylamide photopolymers shows significant differences.

Keywords: Recording materials, photopolymers, holography 


\section{Introduction}

Photopolymers are useful for different applications due to the refractive index variations and relief profiles generated [1-5]. There are many types of photopolymers that may be differentiated by the type of binder, since this component determines to a great extent the choice of monomer, dye and initiator used in the photopolymer. Photopolymers with a hydrophilic binder include hybrid materials made by the sol-gel procedure [6]. Photopolymers with a polyvinylalcohol or gelatin binder and monomers related to acrylamide (AA) are also hydrophilic [7, 8]. All these photopolymers have an undesirable feature, the toxicity of their components and their low environmental compatibility, particularly if we analyze the life cycle of the devices made with these materials and their interaction with the environment. In hydrophobic photopolymers the use of petroleum based solvents during the processes of production is common and it is likely that such solvents will also be used in any recycling or destruction of these materials if they are used in future years for the production of commercial products with a widespread use. However, nowadays the tendency is to limit the use of petroleum based solvents such as chloroform or methanol due to their toxic potential [9]. Hydrophilic photopolymers with AA as the polymerizable monomer are versatile materials for use as holographic recording media [10]. The main drawback of an AAbased photopolymer as far as the environment is concerned is the acrylamide, a substance which has been known to be carcinogenic for many years. Recent investigations confirm the toxic potential of AA [11-13].

In order to develop a new photopolymer with environmental compatibility we used as a base the AA-based photopolymer and "Biophotopol" composition proposed in references [14-15]. 
We have developed a new photopolymer with photochemical and holographic features similar to PVA/AA based but with an improved design from the environmental point of view. This new photopolymer is called "Biophotopol". We have demonstrated some experimental properties of thick layers (1 $\mathrm{mm}$ of thickness) for data holographic storage applications [15]. Thick layers are characterized by low concentrations of monomer and dye [5] in order to obtain high values of physical thickness [16]. Now, in the present work we have analyzed the limit of zero frequency response [17-18] to characterize some different chemical compositions. Using zero frequency recording we can eliminate the influence of monomer diffusion in the material behaviour. In addition, the modulation properties of these materials are in general very different at low spatial frequencies in comparison with the results at the typical range of holographic spatial frequencies. In the limit of zero frequency recording, i.e., uniform exposure of the photosensitive layer, no diffusion processes are produced [18] and we can determined directly many parameters (as opposed to holographic characterization, the data obtained in this case are directly related to the physical properties of the material without the need of nonlinear fitting of multiparametric models) and these values can be used in diffusion models to predict the exact holographic material behavior. Some of these parameters are shrinkage (holes generation), polymerization rate, polymer refractive index and relation between polymerization and recording intensity, etc. The interferometric method proposed has been applied with success to characterize PVA/based materials. In this work we have carried on a complete characterization of Biophotopol materials. In Section 2 we explain the experimental data treatment and we present the equations and concepts used to calculate the material parameters. In Section 3 we introduce the experimental setup and we show the different compositions of Biophotopol tested in order to improve the stability and the optical properties and in 
Section 4 we analyze the results and we have compared this new material based on PVA/NaAO with the results obtained with the AA-based photopolymer. The main conclusions of this work are given in Section 5.

\section{Theoretical data treatment.}

The phase shift between the exposed and non-exposed areas in the transmission interferometric case is due to two different effects: monomer polymerization in the exposed zones and thickness variations when polymerization occurs. Since we want to study separately these two effects, we can use a reflection interferometric setup to analyze the thickness variation during exposition at the zero spatial frequency limit. In this case, the thickness variations of the material $(\Delta d)$ can be expressed as follows:

$$
\Delta d=\frac{\Delta \Phi_{S h r} \lambda \cos \alpha}{4 \pi}
$$

where $\Delta \Phi_{S h r}$ is the phase-shift (in radians) between the exposed and non-exposed zones due to the shrinkage along the whole round-trip forward reflected paths, $\lambda$ and $\alpha$ are the wavelength and the incident angle of the reading beam respectively. Usually, shrinkages around $8 \%$ are reported in the literature for different polymer formulations $[19,20]$. Shrinkage of $0.5 \%$ is defined as the upper limit for commercial availability of a recording medium $[5,21,22]$. Nevertheless, it is important to note there are other cases where relief profiles (higher shrinkage or swelling) are required, i.e. liquid crystal related applications [23].

Once we have obtained the experimental results (in transmission, where both refractive index and volume variations have influence, and in reflection, where only 
volume variations have influence) we can obtain the average refractive index following the same method of reference [18]. The phase shift measured in the transmission case $\left(\Delta \Phi_{t}\right)$ can be described as follows:

$$
\Delta \Phi_{t}=\frac{2 \pi}{\lambda} d(t) \cdot \Delta n \cos \alpha^{\prime}-\Delta \Phi_{S h r}
$$

where $d(\mathrm{t})$ is the new "solid" layer thickness [18], $\quad \alpha^{\prime}$ is the angle inside the material and $\Delta \Phi_{S h r}$ is the phase shift due to the shrinkage and can be expresses as:

$$
\Delta \Phi_{S h r}=\frac{2 \pi}{\lambda} \Delta d\left(n_{d a r k} \cos \alpha^{\prime}-\cos \alpha\right)
$$

where $\Delta d$ is the layer shrinkage. Afterwards, it is necessary to take into account the polymerization of monomer together with the variation of the "hole" volume fraction inside the new "solid" layer [18]. In this sense, it is extremely important to evaluate this last effect if we want to obtain a realistic estimation of the index variation between the exposed and non-exposed zones. Indeed, due to the reduction in the "hole" volume fraction, the refractive index of the new "solid" layer increases largely [18]. Therefore the variation of the refractive index of the "solid" layer represented has been calculated using the following equation:

$$
\Delta n=\frac{\lambda \Delta \Phi_{t} \cos \alpha^{\prime}}{2 \pi d(t)}+\frac{\Delta d}{d(t)}\left(n_{\text {dark }} \cos \alpha^{\prime}-\cos \alpha\right)
$$

This equation can be simplified for small incident angles $\left(\cos \alpha^{\prime}=\cos \alpha=1\right)$. In the case, for the whole layer the refractive index variation $\left(\Delta n_{w}\right)$ can be calculated as follows: 


$$
\Delta n_{w}=\frac{\lambda \Delta \Phi_{t} \cos \alpha^{\prime}}{2 \pi d_{0}}
$$

Along the polymerization process, the holes are expulsed out of the "solid" layer and so its density increases [18].

$$
\phi^{(m)}+\phi^{(p)}+\phi^{(h)}+\phi^{(b)}=1
$$

where $\phi^{(m)}, \phi^{(p)}, \phi^{(h)}$ and $\phi^{(b)}$ are the volume fractions of the monomer, the polymer, the holes and the background (PVA, triethanolamine and dye) respectively. While the collapse of holes will result in a reduction in the overall volume, the total volume fraction is by definition conserved. We can assume that $\phi^{(b)}$ remain constant during the exposition and polymer and holes (on the surface) begin to appear when the monomer starts to polymerize. Therefore we can assume that

$$
\phi^{(m)}+\phi^{(p)}+\phi^{(h)}=\phi_{0}^{(m)}
$$

Where, $\phi_{0}{ }^{(m)}$ is the initial monomer volume fraction.

At zero spatial frequency limit, when diffusion does not take place, the decrease in the monomer concentration depends on the recording intensity, $I$, the dependency of the polymerization and intensity, $\gamma$, initial monomer concentration and the polymerization reaction constant, $K_{R}$.

$$
\phi^{(m)}(t)=\phi_{0}^{(m)} e^{-I^{\gamma} K_{R} t}
$$

We also can assume that the creation of holes and polymer is proportionality produced; thus the volume fraction of holes and polymer may be described by: 


$$
\phi^{(p)}=\alpha \phi^{(h)}
$$

If we introduce eq. (9) in eq. (7), we can obtain the volume fraction of polymer as:

$$
\begin{gathered}
\phi^{(h)}(1+\alpha)=\phi_{0}^{(m)}-\phi^{(m)} \\
\phi^{(h)}(1+\alpha)=\phi_{0}^{(m)}\left(1-e^{-I^{\gamma} K_{R} t}\right)
\end{gathered}
$$

Using eq. (11) it is possible to obtain the hole volume fraction for very long exposure times, when all the monomer has been polymerized. Then the shrinkage $\left(\phi^{(h)}\right)$ remains constant in zero spatial frequency limit.

$$
\phi_{\infty}^{(h)}=\frac{\phi_{0}^{(m)}}{(1+\alpha)}
$$

Now it is possible to obtain a linear dependence of the shrinkage, proportional to the polymer concentration, by the polymerization rate $\left(F_{R}\right)$ defined by many authors [24].

$$
\ln \left(1-\frac{\phi^{(h)}}{\phi_{\infty}^{(h)}}\right)=-I^{\gamma} K_{R} t=-F_{R} t
$$




\section{Experimental setup and composition}

In this Section we focus on the description of the combined setup to obtain real time interferometric measurements while the holographic material is being exposed. First we describe the combined setup with the interferometer in transmission. Then we describe the specific details for the interferometer in reflection.

In reference [18] we show the experimental setup in transmission geometry to measure the phase-shift as a function of the exposure energy.

To generate monomer polymerization we use a wavelength of $532 \mathrm{~nm}$ (at this wavelength the dye presents the maximum absorption), and for the interferometric arm, to generate the interferences pattern we use a He-Ne laser, since the photopolymer does not present any absorption at $633 \mathrm{~nm}$. We have implemented a Young's fringes based two beams interferometer. This interferometer has been successfully applied in the phase-shift characterization of liquid crystal displays (LCDs) [25].

The scheme for the setup to measure the phase-shift in reflection is basically the same as used in reference [26]. With these measurements the thickness variation, thus the shrinkage, can be measure as a function of the exposure energy. Therefore, with this setup it is possible to plot the fringes moving as a function of the exposure time during the polymerization process, and without the influence of monomer diffusion. In this sense, we have verified that once we stop the exposition, the shift in the interference fringes freezes. This indicates that the measurements taken in this setup are not affected by diffusion processes. In the case of the photopolymer studied in this paper we obtained that the exposed zone shrinks with respect to the non-exposed area.

As is detailed in references [14-15], for the monomer substitution it is necessary to use another vinyl monomer that is less toxic than AA. The monomer must be water soluble, it must not evaporate during the preparation of layers and it has to react through a 
radical chain mechanism. The toxicity of sodium acrylate $(\mathrm{NaAO})$ is lower than that of AA [15]. Moreover, different triethanolamine (TEA) concentrations are normally used to obtain different material properties (TEA is a liquid at ambient temperature and plays an important role in the monomer and polymer diffusion during the polymerization process too).

The less toxic component of PVA/AA standard photopolymer is the dye, Yellowish Eosin (YE), nevertheless to obtain a real environment compatibility we have tested less toxic dye [27]. In 1999 Bertolotti and coworkers studied the riboflavin/TEA pair as photoinitiator system in vinyl monomer polymerisation [28]. In the new photopolymer we use the sodium salt 5'-riboflavin monophosphate (PRF) as dye, bearing in mind that this substance is water soluble and exists in the environment, so it is not likely to cause environmental problems.

The solutions, whose compositions can be seen in Table 1, with water as solvent, and PVA as binder $(\mathrm{Mw}=130000$ a.m.u., hydrolysis degree $=87.7 \%)$ are deposited, using the force of gravity over a glass. The glass surface measures $30 \times 40 \mathrm{~cm}^{2}$ and left inside a dark camera where humidity and Temperature are controlled (relative humidity $=40$ $45 \%, \mathrm{~T}=21-23^{\circ} \mathrm{C}$ ) [17]. When large part of the water has evaporated (about 48 hours), the layer has enough mechanical resistance and it can be cut without deformation. As crosslinker we have used N, N'-Methylene-Bis-Acrylamide (BMA) to study the mixture with $\mathrm{NaAO}$. The presence of BMA in the formulation of biophotopol is optional and not essential to the main features of the photopolymer. Furthermore, the toxicity of BMA is clearly lower than AA. In this work we have characterized five new compositions. We have tested photopolymers with different TEA concentrations, with different concentration of BMA, and different dye. Composition A is directly derived from the previous works where biophotopol composition is optimized to achieve thick layers 
(around $1 \mathrm{~mm}$ thick). Since we have found weak modulation of layer type A, we have introduced BMA as crosslinker in compositions B and C. To increase the differences between exposed and non exposed zones we have also introduce more $\mathrm{NaAO}$ in compositions B and D. To substitute YE we have presented composition E. The standard compositions used in previous works are compositions $F$ and G (PVA/AA based materials without and with crosslinker). The thickness of the samples is around $(100 \mu \mathrm{m})$.

\section{Results and discussion}

In this section we present the experimental data obtained for different material compositions and different thicknesses using both experimental setups (Section 4.1 for transmission and 4.2 for reflection). Then on Section 4.3 we have obtained some interesting parameters to understand the optic material behavior. We also have compared the results with PVA/AA based materials.

\subsection{Transmission experiments}

In transmission analysis we can observe the effects of the refractive index changes and thickness variation during polymerization. The results for the new five compositions are presented in Fig. 1. As can be seen the changes in the phase between exposed and nonexposed zones for compositions $\mathrm{A}$ and $\mathrm{E}$ are very weak (around $60^{\circ}$ ), therefore the polymerization rate is small. As the velocity of the reaction is very slow, after $1000 \mathrm{~s}$ the material continues changing in compositions A, E and D (compositions without crosslinker). Compositions B and C (compositions with crosslinker) present very similar behavior in transmission analysis. In these compositions after $400 \mathrm{~s}$ the reaction stops and the layers are in saturation state (where the phase shift does not change with the 
illumination). This fact indicates that all the monomer is consumed in the illuminated zones. Moreover, it is important to note that the phase shift is lower than $360^{\circ}$ and this phase depth is required for many diffractive applications (i.e. to fabricate lens). To achieve higher phase depth the thickness of the layer should be increased. Furthermore it is important to remark the influence of the TEA concentration when comparing layers $\mathrm{B}$ and D. These two compositions have the same quantity of $\mathrm{NaAO}$, besides composition B has crosslinker but the phase shift achieved is similar. This fact can be explained by the differences in TEA concentration. As composition B have low composition of TEA the NaAO have more difficulties to be solved and can crystallize during the drying and recording process. In Fig. 1.b the previous results using PVA/AA based materials are depicted. As can be seen the results obtained with Biophotopol are similar to results obtained with composition $\mathrm{F}$ (without crosslinker). The results obtained with composition G (with BMA as crosslinker) are clearly better than the one with the Biophotopol. The energetic sensitivity is smaller and the phase shift achieved is around $450^{\circ}$.

\subsection{Reflection experiments}

Using reflection experiments we can obtain the thickness variation for different compositions (with and without crosslinker, with different monomer and TEA concentrations), at zero frequency. Once obtained the phase shift between exposed and non exposed zones as a function of the exposure with the reflection interferometer, the shrinkage of the layer during exposure can be directly calculated (see section 4.3.1). In Fig. 2.a we present the results obtained in reflection analysis for $\mathrm{NaAO}$ based compositions (A, B, C, D and E). As can be seen the behavior is similar to the one obtained in transmission analysis. It is important to note that compositions $\mathrm{B}, \mathrm{C}$ and $\mathrm{D}$ 
present similar behavior. These layers can achieve around $3000^{\circ}$ of phase shift. The composition E, with PRF as a dye, the phase shift is around $300^{\circ}$ due to the poor solubility inside the photopolymer and the low monomer reaction velocity. This problem should be solved in order to obtain competitive thin layers. In Fig. 2.b we have introduced the results of the PVA/AA based materials. When the new chemical formulations, Fig.2.a, are compared to the "standard" materials, Fig. 2.b, it can be seen the similar behaviour of $\mathrm{B}, \mathrm{C}, \mathrm{D}$ and $\mathrm{F}$ compositions.

\subsection{Parameters estimation}

Taking into account the experimental results of the last two sections we want to obtain some basic parameters of the new Biophotopol compositions for thin layers (around 100 $\mu \mathrm{m}$ of thickness).

\subsubsection{Shrinkage}

Shrinkage during polymerization is provided directly from Eq. (1) applied to Fig. 2. Shrinkage of $0.5 \%$ is defined as the upper limit for commercial availability of a holographic recording medium. In particular, in the case of holographic grating recorded, molecular migration reduces the shrinkage. This reduction increases with the spatial frequency and with the diffusion velocity.

In Fig. 3 we have presented the results obtained in reflection analysis for $\mathrm{NaAO}$ based compositions (A, B, C, D, and E). We can observe that the maximum shrinkage is around $3 \%$ for compositions $\mathrm{B}, \mathrm{C}$ and $\mathrm{D}$. This shrinkage is enough to store reflection diffractive elements using the relief variations [29]. On the other hand to use these materials as holographic memories the shrinkage should be reduced. As can be deduced from this and last figures compositions B, C and D present similar behavior. The 
composition E, with PRF as a dye, the shrinkage is around $0.25 \mu \mathrm{m}$, nevertheless we cannot extract any significant information because the dye crystallizes during exposition.

\subsubsection{Polymerization rate}

The shrinkage is due to the layer compaction during polymerization. To obtain the magnitude of the velocity of the chemical reaction, the polymerization rate, $F_{R}$, we have used Eq. (13). In Fig. 4 we have depicted the results for some compositions obtained by the fitting based on Eq. (13). The results show us different values of $F_{R}$ for each composition. The results of $F_{R}$ for each composition are presented in Table 2. The correlation value of the fittings indicates the good agreement between theory and experiments. In addition the compositions $\mathrm{A}$ and $\mathrm{D}$ presents similar values of $F_{R}$ because the only variation is the monomer concentration. We have omitted the fitting for the composition $\mathrm{F}$ due to the poor repeatability. The problem of this type of layers is that the solubility of PRF inside the layer depends on dramatically of the drying conditions.

The influence of the BMA, crosslinker, in the increasing of polymerization rate has been demonstrated. Furthermore standard compositions based on PVA/AA present higher values of polymerization rate. On the other hand the layers based on PVA/NaAO present competitive values of $F_{R}$ and better environmental compatibility.

\subsection{3 $\gamma$, relation between exposure intensity and polymerization rate}

The determination of the dependence of the polymerization rate to the exposure intensity is a traditional problem associated to the photopolymerizable systems [23]. Parameter, $\gamma$ (which govern, the relation between recording intensity and polymerization 
rate, see Eq. (8)) can be associated to the viscosity of photopolymer system. In this sense values of $\gamma$ around 0.5 are associated to high fluid systems and for very dry systems values are near to 1 . We have estimated the value of $\gamma$ using material response at zero spatial frequency limit. To achieve this goal we have exposed the material to two different exposure intensities 0.5 and $1 \mathrm{~mW} / \mathrm{cm}^{2}$ for composition type A and we have calculated the polymerization rate value in each experiment using Eq. (13). The comparative result between the two exposures intensities are presented in Fig. 5. In this moment it is easy to obtain the value of $\gamma$. In our case the value obtained is 0.48 , this value is agreed with the value of 0.5 , usually assumed for photopolymers with low viscosity [30]. It is important to note that for low values of the parameter $\gamma$ the nonlinearity is increased.

\subsubsection{Whole layer the refractive index variation $\left(\Delta n_{w}\right)$ and average Polymer}

\section{refractive index $\left(n_{p}\right)$.}

Taking into account transmission results detailed in section 4.1 and Eq. (5) we obtain the variations of the refractive index between exposed and non-exposed zones for the whole layer (Table 3). The saturation value of the refractive index modulation must be higher (around 30\% for PVA/AA based materials [18]) than the typical values of refractive index modulation obtained in the holographic range (an exposure intensity of $5 \mathrm{~mW} / \mathrm{cm}^{2}$ ), where the spatial frequencies are around 1000 lines $/ \mathrm{mm}$. In order to explain these results the main hypothesis is that at very low spatial frequencies, in particular in the zero spatial frequency limit, non-local polymerization does not take place [31]; therefore, polymer chains can become longer without invading the non-exposed zones and so the refractive index also increases. 
Now we want to complete the study for PVA/NaAO based materials. Since diffusion does not exist at the zero spatial frequency limit, and according Lorenz-Lorenz equations [18], we can estimate the average polymer refractive index in the material [18]. In order to calculate an approximation of this refractive index, we will assume that when exposition finishes, all the monomer have polymerized. In this sense, it is important to obtain a precise estimation of the initial volume fraction of the monomer present in the "dry" layer [31-32]. This volume fraction can be estimated if we measure the quantity of water evaporated during the drying process. It is interesting to note that after the drying process only very small portion of the initial water remains inside the layer. In our case, 48 hours after the layer deposition, the initial monomer volume fraction reaches the values presented in table 4 .

After 2000 seconds of exposition, we can assume the final polymer volume fraction is similar to the initial monomer volume fraction $\left(\phi_{1 f}{ }^{(p)} \approx \phi_{1 i}{ }^{(m)}\right)$. In addition, the effect of holes generation and collapse described in reference [30] does not affect when we study the zero spatial frequency limit due to the uniformity in the exposition [18]. That is, we can obtain the polymer average refractive index using equation Lorenz-Lorenz as follows:

$$
\Delta n_{w}=\frac{\left(n_{\text {dark }}^{2}+2\right)^{2}}{6 n_{\text {dark }}}\left[-\left(\frac{n_{m}^{2}-1}{n_{m}^{2}+2}-\frac{n_{b}^{2}-1}{n_{b}^{2}+2}\right) \phi_{1}^{(m)}+\left(\frac{n_{p}^{2}-1}{n_{p}^{2}+2}-\frac{n_{b}^{2}-1}{n_{b}^{2}+2}\right) \phi_{1}^{(p)}\right]
$$

where $n_{\text {dark }}$ is the average refractive index of the material before exposure, $n_{p}$ is the polymer refractive index, $n_{m}$ is the monomer refractive index, $n_{b}$ is the binder refractive index (mixture of PVA, water and TEA) and $\phi_{1}{ }^{(i)}$ is the volume fraction variation of the $\mathrm{i}^{\text {th }}$ component. In the PVA/NaAO based material used in this work, the different refractive indexes have the following values: $n_{m}$ (the values of monomer refractive 
index provided by the method based on the refractometer measurements using water solutions are: 1.486 and 1.565 for $\mathrm{AA}$ and $\mathrm{NaAO}$ respectively), $n_{b}$ depends on TEA concentration (1.370 for composition A, 1.476 for compositions B and C and 1.474 for compositon D), $n_{\text {dark }}$ depends also on the material composition and the drying process and is measured using an Abbe refractometer. In addition the values were obtained using the Lorentz-Lorenz equation and the method described in references [30] and [31]. The calculations are based on refractometer measurements using water solutions and the "dry" layer.

And according to Eq. (14), we can obtain the following approximate equation:

$$
\frac{n_{p}^{2}-1}{n_{p}^{2}+2}=\frac{1}{\phi_{f}^{(p)}}\left[\frac{6 n_{\text {dark }}}{\left(n_{\text {dark }}^{2}+2\right)^{2}} n_{w}+\left(\frac{n_{m}^{2}-1}{n_{m}^{2}+2}-\frac{n_{b}^{2}-1}{n_{b}^{2}+2}\right) \phi_{i}^{(m)}\right]+\frac{n_{b}^{2}-1}{n_{b}^{2}+2}
$$

Results provided by Eq. (15) are presented in Table 5 (we have omitted composition $\mathrm{G}$ due to its different behavior). These values for $n_{p}$ are important when are compared with the monomers ones; i.e. the refractive index of acrylamide is clearly lower than the $\mathrm{NaAO}$ refractive index. To determinate these values we have followed the steps indicated in Ref. [30-31]. Furthermore we want to remark that the main important point is to compare $n_{m}$ with $n_{p}$ and these parameters with $n_{b}$ (Eq. 14). In this sense the importance of crosslinker to increase $n_{p}$ has been demonstrated using the zero frequency technique. The low value obtained for composition $\mathrm{C}$ can be explained by the precipitation of part of BMA during the drying process. To improve this composition the concentration of TEA should be increased like composition D and compositions presented in ref. [30]. As can be seen the value of $n_{p}$ for composition A is lower than the composition $\mathrm{D}$, this fact can be understand by the presence of higher concentration of monomer: this provide longer polymer chains (larger values of refractive index). 
It is important to mention that our estimations of the polymer average refractive index are based on experimental direct measurements whereas in holographic applications previous determinations of many parameters are needed, that is, estimations of the polymer average refractive indexes in holography are always indirect. In this sense, we must not forget that according to our hypothesis (see the beginning of this subsection and reference [18]), the values obtained for the zero spatial frequency limit are always upper limits to the values for other spatial frequencies.

\section{Conclusions}

In this work, we have proposed different possibilities to achieve thin photopolymer layers with high environmental compatibility. Using direct measurements of the phase shift and the shrinkage of photopolymeric layers based on PVA/NaAO (for five different chemical compositions) we have estimated different material properties. The main advantage of this method is the absence of monomer diffusion effects in the experiments carried out. We have obtained values of the shrinkage between $1 \%$ and $3 \%$ in the layers analyzed. These values are promising so as to obtain relief structures for liquid crystal applications, but, on the other hand, are considerably high for holographic memories applications. Furthermore we have obtained the values for the polymerization rates and the relation between polymerization and exposure intensity. The results present good agreement with theoretical predictions. Eventually we have compared the results with the typical materials based in PVA/AA and we have shown that sodium acrylate permits to obtain less toxic photopolymer thin layers available for very low spatial frequency applications. In order to achieve "greener" layers the optimization of the incorporation of RF as a dye should be done. One possible solution is the decreasing in the RF concentration and the increase of the layer thickness, looking for an 
intermediate step between thin and thick layers [15]. On the second part of the paper we have obtained quantitative information of the materials based on PVA/NaAO. We have demonstrated that the values of $K_{R}$ are lower for this family of materials and we have obtained a value of $\gamma$ close to the theoretical assumptions [29]. In the last part we have obtained significant differences between the refractive indexes of $\mathrm{AA}$ and $\mathrm{NaAO}$ based materials due to the differences in the $n_{m}$ parameter. All these parameters should be taking into account in order to design holographic diffusion model to predict the behavior of this family of photopolymers with high environmental compatibility. In our opinion the viability of PVA/NaAO based photopolymers for diffractive and holographic application has been demonstrated.

\section{Acknowledgements}

This work was supported by the "Ministerio de Edicación y Ciencia" (Spain) under projects projects FIS2008-05856-C02-01 and FIS2008-05856-C02-02, by the Generalitat Valenciana (projects ACOMP/2009/150 and ACOMP/2009/160). 


\section{References}

1. J. Neumann, K. S. Wieking and D. Kip "Direct Laser Writing of Surface Reliefs in Dry, Self-Developing Photopolymer Films," Appl. Opt. 38, 5418-5421 (1999).

2. X.T.Li, A. Natansohn, P. Rochon "Photoinduced liquid crystal alignment based on a surface relief grating in an assembled cell” Appl.Phys.Lett. 74(25) 3791-3793 A (1999). 3. A. Márquez, S. Gallego, M. Ortuño. E. Fernández, M. L. Álvarez, A. Beléndez, I. Pascual "Generation of diffractive optical elements onto a photopolymer using a liquid crystal display” SPIE, Optical Modelling and Design, Vol. 7717, 77170 (2010).

4. M. D. Lechner, "Photopolymers for optical memories and waveguides" Electron. Prop. Polym. Relat. Compd. 63, 301-308 (1985).

5. H. J. Coufal, D. Psaltis, and G. T. Sincerbox, eds., Holographic Data Storage, Springer Series in Optical Sciences, (Springer-Verlag, Berlin, 2000).

6. P. Cheben, and M. L. Calvo "A Photopolymerizable Glass with Diffraction Efficiency Near 100\% for Holographic Storage" Appl. Phys. Lett. 78, 1490-1492 (2001).

7. V. Weiss, E. Millul, and A. A. Friesem, "Photopolymeric holographic recording media: In-situ and realtime characterization" Proc. SPIE 2688, 11-21 (1996).

8. L. Feng, Y. Jin, C. Xia, H. Lan, and K. Wang, "Study on a high performance redsensitive photopolymer-gelatin holographic recording material" J. Sichuan University $38,773-775$ (2001). 
9. F. Pellaschiar, "Reduction of the utilization of VOC and their atmospheric emissions. Development of legislation and research in the use of less toxic solvents and reduced environmental impact” Eur. Coat. J. 81, 22-28 (2005).

10. S. Sugawara, K. Murase, and T. Kitayama, "Holographic recording by dyesensitized photopolymerization of acrylamide” Appl. Opt., 14, 378-382 (1975).

11. K. Hashimoto, and W. N. Aldridge, "Biochemical studies on acrylamide, a neurotoxic agent” Biochem. Pharmacol. 19, 2591-2604 (1970).

12. F. Mendel, "Chemistry, biochemistry, and safety of acrylamide. A review" J. Agric. Food. Chem. 51, 4504-4526 (2003).

13. A. L. Lipman, Safety of xanthene dyes according to the U.S. Food and Drug Administration, ACS Symposium Series, American Chemical Society, USA, [LightActivated Pest Control 616, 34-53 (1995)].

14. M. Ortuño, S. Gallego, C. Neipp, A. Márquez, A. Beléndez, and I. Pascual, "Material de registro holográfico medioambientalmente compatible basado en acrilatos metálicos" Patent number ES 200503113 (2005).

15. M. Ortuño, E. Fernández, S. Gallego, A. Beléndez, and I. Pascual, "New photopolymer holographic recording material with sustainable design," Opt. Express 15, $12425-12435$ (2007).

16. S. Gallego, M. Ortuño, C. Neipp, A. Márquez, A. Beléndez, I. Pascual, J. V. Kelly, J. T. Sheridan, "Physical and effective optical thickness of the holographic diffraction gratings recorded in photopolymers" Optics Express 13, 3543-3557 (2005).

17. S. Gallego, A. Márquez, D. Méndez, S. Marini, A. Beléndez, and I. Pascual, "Spatial phase modulation-based study of PVA/AA photopolymers in the low spatial frequency range," Appl. Opt. 48, 4403-4413 (2009). 
18. S. Gallego, A. Márquez, D. Méndez, C. Neipp, M. Ortuño, M. L. Alvarez, E. Fernandez, A. Beléndez, and I. Pascual, "Real-time interferometric characterization of a polyvinyl alcohol based photopolymer at the zero spatial frequency limit," Appl. Opt. 46(30), 7506-7512 (2007).

19. J.E. Dietz, N.A. Peppas, "Reaction kinetics and chemical changes during polymerization of multifunctional (meth)acrylates for the production of highly crosslinked polymers used in information storage systems", Polymer, 38 (15), 37673781, (1997).

20. T. Endo, F. Sanda, "Ring-opening polymerization, anionic (with expansion in volume)", Polymeric Materials Encyclopedia, 10, CRC Press, Inc., 7550-7554, (1996).

21. G. Ramos, A. Álvarez-Herrero, T. Belenguer, F. del Monte, and D. Levy, "Shrinkage Control in a Photopolymerizable Hybrid Solgel Material for Holographic Recording," Appl. Opt. 43, 4018-4024 (2004).

22. L. Dhar, M. G. Schones, T. L. Wysocki, H. Bair, M. Schilling, and C. Boyd, “Temperature-induced changes in photopolymervolume holograms," Appl. Phys. Lett. 73, 1337-1339 1998.

23. I. Naydenova, E. Mihaylova, S. Martin, and V. Toal, "Holographic patterning of acrylamide-based photopolymer surface," Opt. Express 13, 4878-4889 (2005)

24. C. Neipp, A. Beléndez, J. T. Sheridan, J. V. Kelly, F. T. O’Neill, S. Gallego, M. Ortuño and I. Pascual "Non-local polymerization driven diffusion based model: general dependence of the polymerization rate to the exposure intensity" Optics Express 11, 1876-1886 (2003).

25. A. Bergeron, J. Gauvin, F. Gagnon, D. Gingras, H. H. Arsenault and M. Doucet, "Phase calibration and applications of a liquid-crystal spatial light modulator", Appl. Opt. 34, 5133-5139 (1995). 
26. S. Gallego, A. Márquez, D. Méndez, M. Ortuño, C. Neipp, E. Fernández, I. Pascual, and A. Beléndez, "Analysis of PVA/AA based photopolymers at the zero spatial frequency limit using interferometric methods," Appl. Opt. 47, (2008)

27. Ortuño M., Márquez A., Gallego S., Neipp C., Fernández E. "pyrromethene dye and non-redox initiator system in a hydrophilic binder photopolymer" Optical Materials 29, $1-4(2007)$.

28. S. G. Bertolotti, C. M. Previtali, "Riboflavin/Triethanolamine as Photoinitiator System of Vinyl Polymerization- A Mechanistic Study by Laser Flash Photolysis" Macromolecules 32, 2920-2924 (1999).

29. K. Pavani, I. Naydenova, S. Martin, R. Jallapuram, R. G. Howard, V. Toal, "Electro-optical switching of liquid crystal diffraction gratings by using surface relief effect in the photopolymer.” Opt. Comm. 273, 367-369 (2007)

30. G. Zaho and P. Moroulis, "Extension of diffusion model of holograhic photopolymer", Optics Communications 15, 528-532 (1995).

31. J.V. Kelly, M.G. Gleeson, C. E. Close, F. O’Neill, J. T. Sheridan, S. Gallego, C. Neipp, "Temporal analysis of grating formation in photopolymer using the nonlocal polymerization-driven diffusion model” Opt. Express 13, 6990-7004 (2005).

32. S. Gallego, PhD Thesis "Modelización del comportamiento holográfico de un fotopolímero de polivinilalcohol/acrilamida” Univertity of Alicante (2005). 


\section{TABLE CAPTIONS}

Table 1. Solution compositions and thickness

Table 2. Polymerization rates $\left(F_{R}\right)$ and correlation parameter $\mathrm{R}^{2}$ for the fittings obtained for each chemical composition.

Table 3. Refractive index variation, $\Delta n_{w}$, for each composition and saturated phase depth in transmission, $\Delta \Phi_{t}$.

Table 4. Initial monomer volume fraction for analyzed compositions.

Table 5. Polymer refractive index, $\boldsymbol{n}_{\boldsymbol{p}}$, and binder refractive index, $\boldsymbol{n}_{\boldsymbol{b}}$, for each composition based on Biophotopol. 


\section{FIGURE CAPITONS}

Fig. 1. Phase shift as a function of the exposure time in transmission experiments for photopolymer compositions a) A, B, C, D and E Biophotopol derived compositions; b) F and G (PVA/AA) based materials.

Fig. 2. Phase shift as a function of the exposure time for photopolymer compositions in reflection experiments for photopolymer compositions: a) A, B, C, D and E Biophotopol derived compositions; b) F and G (PVA/AA) based materials

Fig. 3. Shrinkages as a function of the exposure time for photopolymer compositions A, $\mathrm{B}, \mathrm{C}, \mathrm{D}, \mathrm{E}$, and $\mathrm{F}$ in reflection experiments.

Fig. 4. Fittings derived from Eq. 13 based on reflection experiments.

Fig. 5. Fittings derived from Eq. 13 based for different exposure intensities (0.5 $\mathrm{mW} / \mathrm{cm}^{2}$ and $1 \mathrm{~mW} / \mathrm{cm}^{2}$ ). 


\section{TABLES}

\section{TABLE 1}

\begin{tabular}{|c|c|c|c|c|c|c|c|}
\hline $\begin{array}{c}\text { Composition } \\
\text { type }\end{array}$ & A & B & C & D & $E$ & $F$ & $\mathbf{G}$ \\
\hline $\begin{array}{c}\mathrm{NaAO} \\
\text { (a) }\end{array}$ & 0.75 & 1.5 & 0.75 & 1.5 & 0.75 & ----- & ------- \\
\hline $\begin{array}{l}\mathrm{H}_{2} \mathrm{O} \\
(\mathrm{ml})\end{array}$ & 12.5 & 12.5 & 12.5 & 12.5 & 12.5 & 14.5 & 14.5 \\
\hline $\begin{array}{l}\text { TEA } \\
\text { (ml) }\end{array}$ & 1.25 & 1.5 & 1.5 & 3 & 3 & 1.25 & 2.3 \\
\hline $\begin{array}{l}\text { PVA (ml) } \\
(15 \% \mathrm{w} / \mathrm{v})\end{array}$ & 12.5 & 12.5 & 12.5 & 12.5 & 12.5 & 12.5 & 12.5 \\
\hline $\begin{array}{c}\text { YE }(0.8 \% \\
\text { w/v) } \\
(\mathrm{ml})\end{array}$ & 0.6 & 0.6 & 0.6 & 0.6 & - $^{------}$ & 0.6 & 0.6 \\
\hline $\begin{array}{l}\text { PRF } \\
\text { (g) }\end{array}$ & ------- & ------- & ------ & $\begin{array}{l}----- \\
- \\
\end{array}$ & 0.14 & ------- & ------ \\
\hline $\begin{array}{c}\text { BMA } \\
\text { (g) }\end{array}$ & ------ & 0.1 & 0.2 & ------- & ------- & ------- & 0.25 \\
\hline $\begin{array}{l}\text { AA } \\
\text { (g) }\end{array}$ & ------- & ------ & ------- & ------ & ------- & 0.96 & 0.84 \\
\hline $\begin{array}{l}\text { Thickness } \\
\pm 4(\mu \mathrm{m})\end{array}$ & 105 & 100 & 97 & 107 & 106 & 100 & 102 \\
\hline
\end{tabular}


TABLE 2

\begin{tabular}{|c|c|c|c|c|c|c|}
\hline $\begin{array}{c}\text { Composition } \\
\text { type }\end{array}$ & A & B & C & D & F & G \\
\hline$F_{R} \times 10^{-3}\left(s^{-1}\right)$ & 3.2 & 3.6 & 4.1 & 3.3 & 5.8 & 7.0 \\
\hline $\mathbf{R}^{2}$ & 0.999 & 0.993 & 0.992 & 0.994 & 0.997 & 0.994 \\
\hline
\end{tabular}

TABLE 3

\begin{tabular}{|c|c|c|c|c|c|c|c|}
\hline $\begin{array}{c}\text { Composition } \\
\text { type }\end{array}$ & A & B & C & D & E & F & G \\
\hline$\Delta \Phi_{t}$ & 90 & 280 & 260 & 290 & 80 & 300 & 500 \\
\hline$\Delta n_{w} \times 10^{-3}$ & 1.49 & 4.86 & 4.66 & 4.71 & 1.31 & 5.21 & 8.51 \\
\hline
\end{tabular}

TABLE 4

\begin{tabular}{|c|c|c|c|c|c|c|}
\hline $\begin{array}{c}\text { Composition } \\
\text { type }\end{array}$ & A & B & C & D & F & G \\
\hline $\begin{array}{c}\text { Initial Monomer } \\
\text { volume Fraction }\end{array}$ & 0.13 & 0.15 & 0.25 & 0.17 & 0.20 & 0.22 \\
\hline
\end{tabular}

Table 5

\begin{tabular}{|c|c|c|c|c|c|}
\hline $\begin{array}{c}\text { Composition } \\
\text { type }\end{array}$ & $\mathrm{A}$ & $\mathrm{B}$ & $\mathrm{C}$ & $\mathrm{D}$ & $\mathrm{E}$ \\
\hline $\boldsymbol{n}_{\boldsymbol{p}}$ & 1.578 & 1.603 & 1.585 & 1.595 & 1.51 \\
\hline $\boldsymbol{n}_{\boldsymbol{b}}$ & 1.470 & 1.476 & 1.476 & 1.474 & 1.470 \\
\hline
\end{tabular}




\section{FIGURES}

FIGURE 1
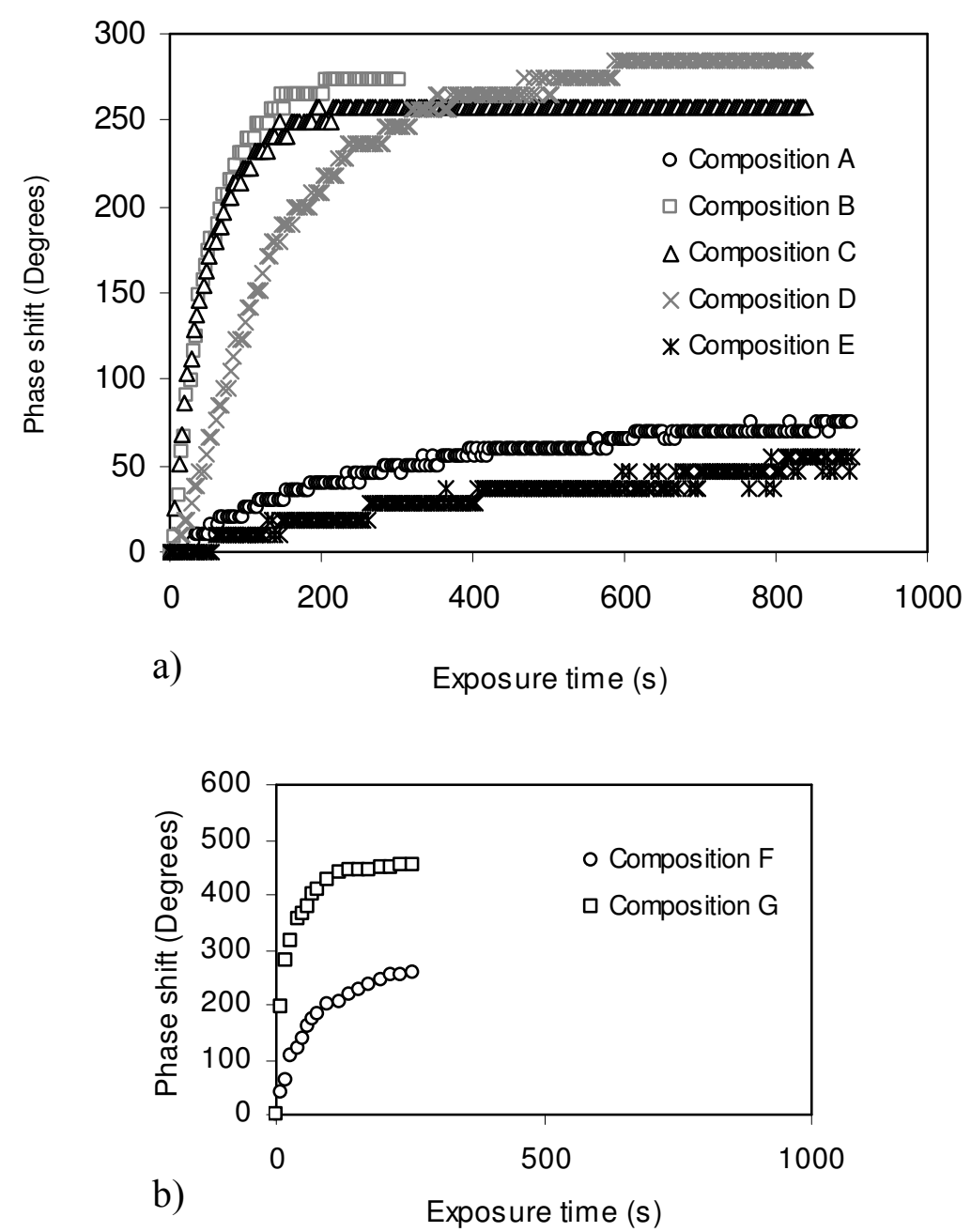
FIGURE 2
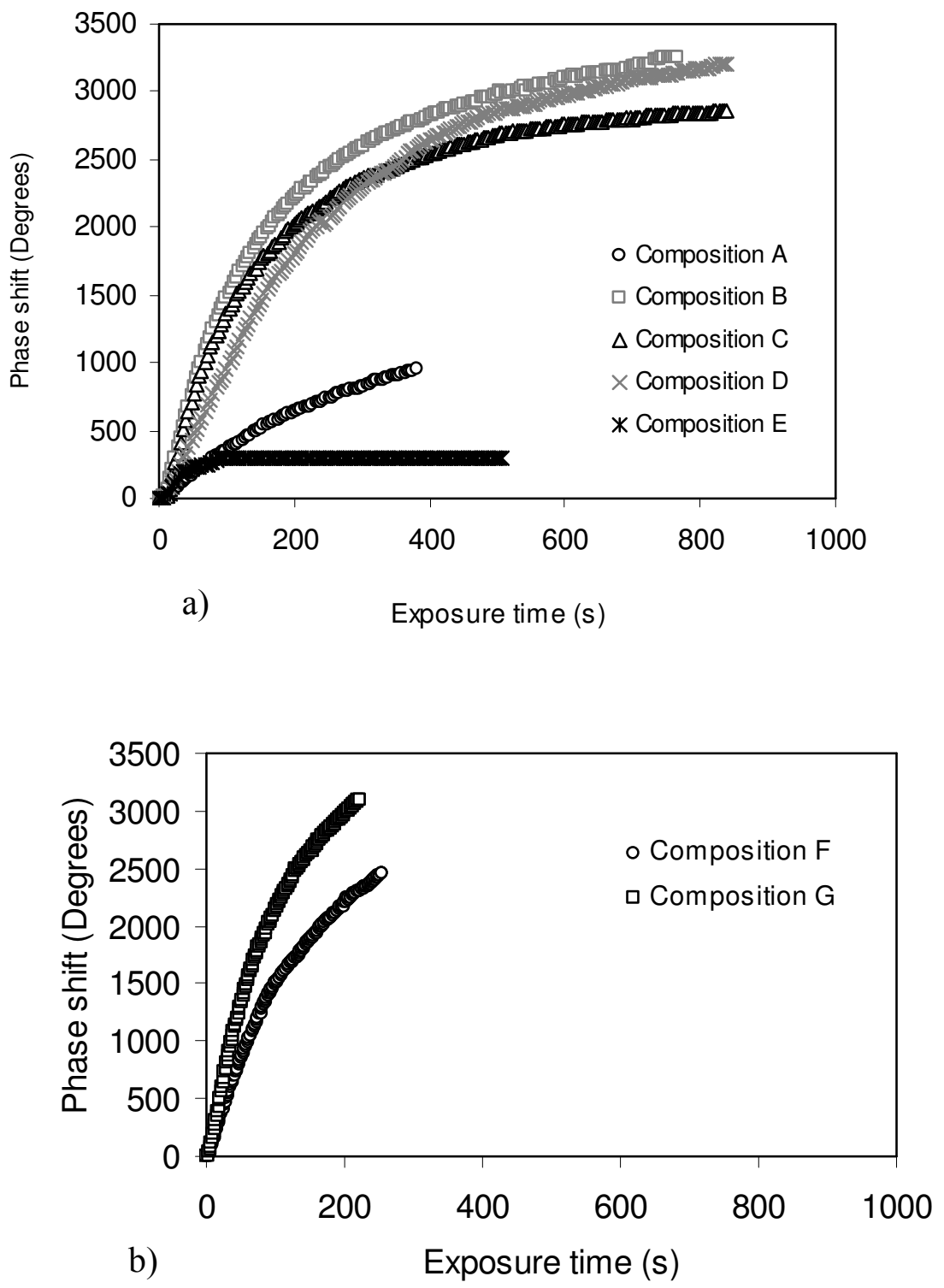
FIGURE 3

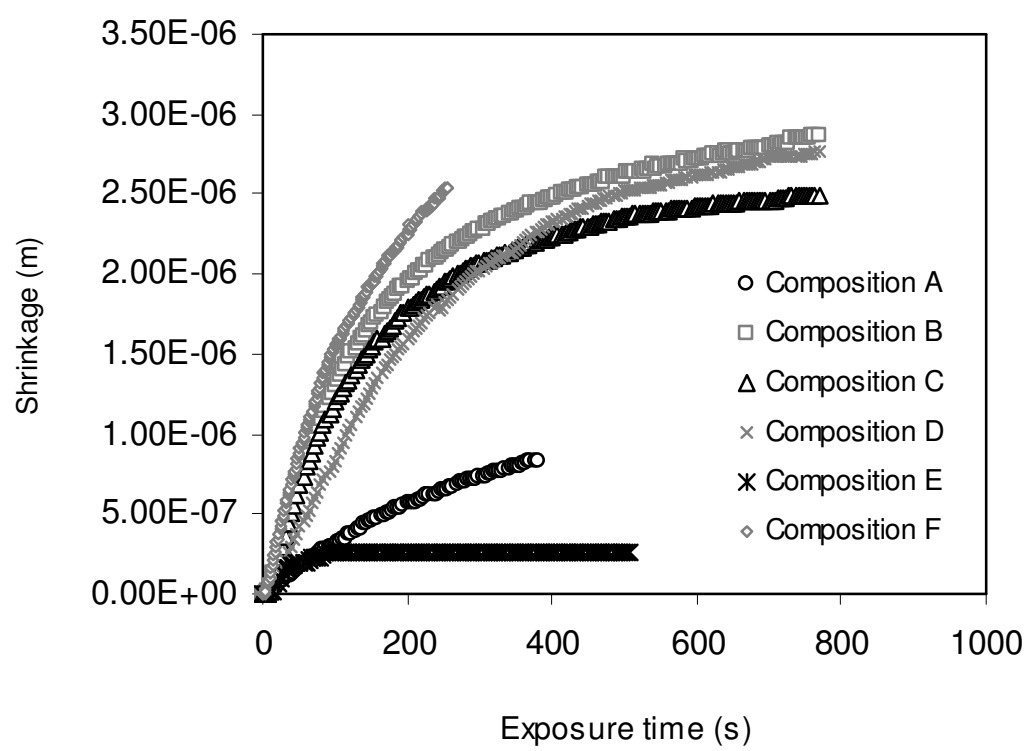


FIGURE 4

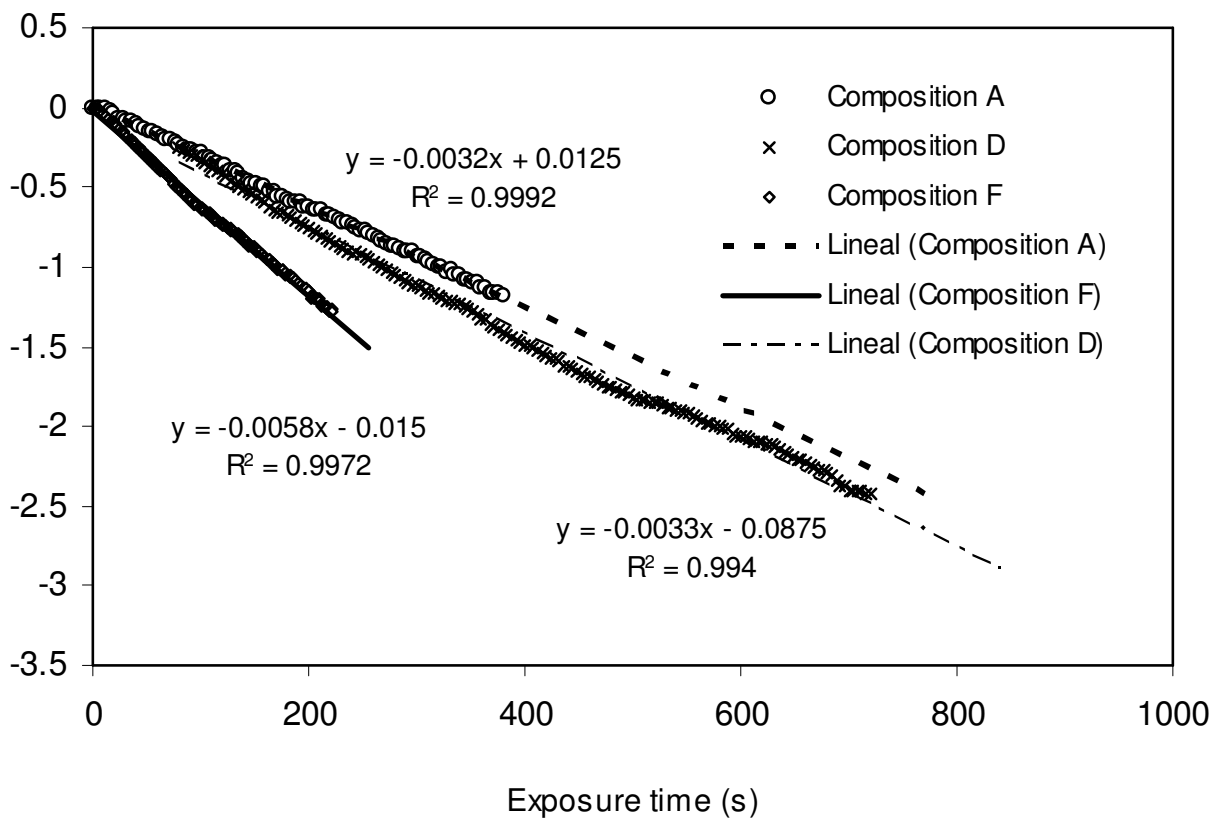


FIGURE 5

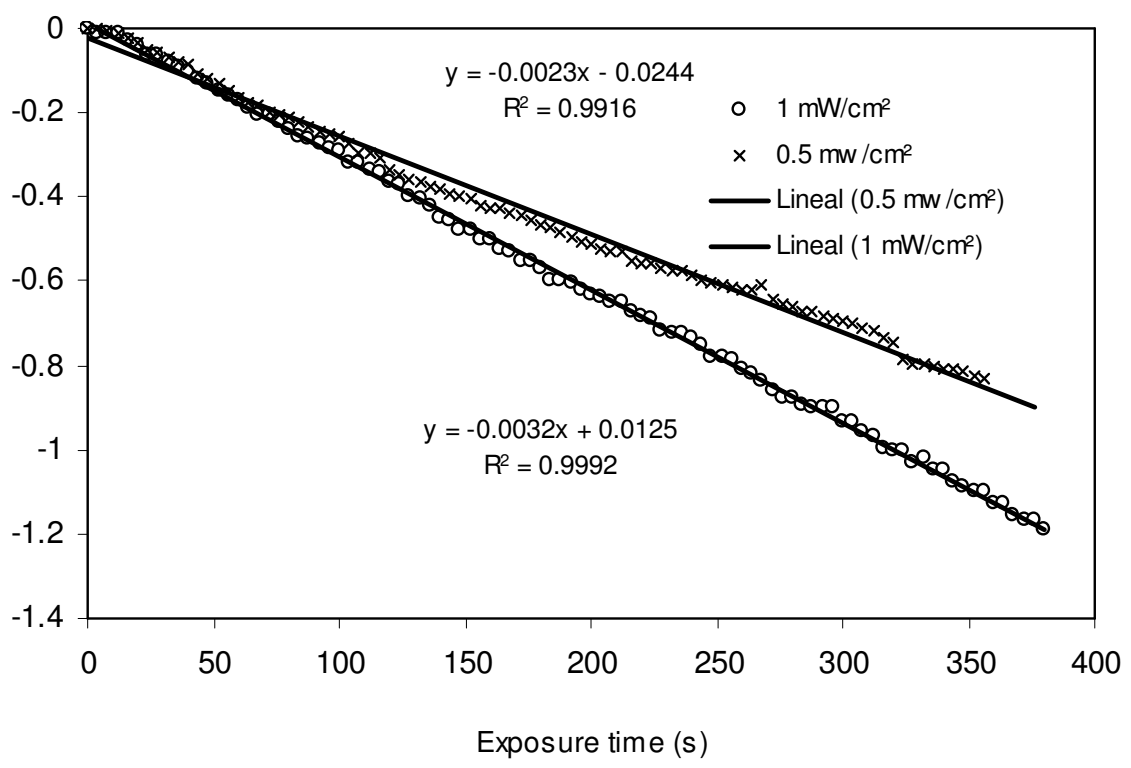

